\title{
Achieving per-flow and weighted fairness for uplink and downlink in IEEE 802.11 WLANs
}

\author{
Chiapin Wang
}

\begin{abstract}
In this article, we investigate a fairness issue between uplink and downlink flows in IEEE 802.11 Wireless Local Area Networks (WLANs). We propose a cross-layer adaptive algorithm which dynamically adjusts the minimum contention window size of access point according to the amount of downlink users and channel conditions to achieve per-flow fairness. In case that uplink and downlink transmissions are with different bandwidth demands for various applications, our algorithm can efficiently find the optimal minimum contention window size which provides weighted fairness based on their resource requirements. The simulation results demonstrate that our scheme can effectively provide both per-flow fairness and weighted fairness in a varying WLAN environment.
\end{abstract}

Keywords: Fairness, IEEE 802.11 WLAN, MAC contention control, Bandwidth allocation

\section{Introduction}

In recent years, IEEE 802.11 Wireless Local Area Networks (WLAN) [1,2] have become increasingly popular with the wide deployment of infrastructures and the prevalence of mobile/handheld devices. Mobile users over WLAN now can access various broadband and real-time services, e.g., video streaming, peer-to-peer applications, Internet protocol television, and Voice over IP. In general, IEEE 802.11 WLANs employ an infrastructure mode in which an access point (AP) acts as a bridge for exchanging two-direction data traffic, i.e., downlink and uplink, between wireless and wired domains. "Downlink" refers to a traffic flow transmitted from AP to a mobile device, while "uplink" refers to a flow with a reverse direction. The 802.11 medium access control (MAC) layer employs a contention-based channel access mechanism, named distributed coordination function (DCF) for its distributed and simple manner. With DCF, all 802.11 nodes with packets to send including AP and mobile stations generally have the same channel-access probabilities. Since AP is responsible for all the deliveries of downlink flows, therefore, the total transmission opportunities of downlink flows will be equal to $1 /(M+1)$ where $M$ is the number of stations. However, such the bandwidth allocation between uplink and downlink flows may not match the user

Correspondence: chiapin@ntnu.edu.tw

Department of Applied Electronic Technology, National Taiwan Normal University, Taipei, Taiwan behavior in real situations while the traffic load of downlink generally is much heavier than that of uplink. The unfairness problem between uplink and downlink can particularly be serious when the amounts of downlink flows increase or the traffic load of downlink is much heavier than that of uplink.

In order to provide fair channel utilization between uplink and downlink, AP and mobile stations should be granted suitable transmission opportunities based on their bandwidth demands. In this article, we propose a cross-layer adaptive algorithm which dynamically adjusts the minimum contention window size of AP based on the amount of downlink flows to achieve per-flow fairness. In case that uplink and downlink transmissions are under diverse channel conditions, our algorithm can also efficiently find the optimal contention window size to provides fairness according to channel conditions while the channel utilization can be affected by not only the amount of contending flows, but also the link qualities, i.e., bit error rate (BER). Furthermore, if uplink and downlink flows are with different bandwidth demands for various applications, our algorithm can adaptively tune the contention window size to provide weighted fairness based on their resource requirements. The contribution of this article is that we present a cognitive algorithm based on a cross-layer design which can sense the changes of wireless environments (e.g., the number of flows, channel conditions, and bandwidth demands),

\section{穴 Springer}

(c) 2012 Wang; licensee Springer. This is an Open Access article distributed under the terms of the Creative Commons Attribution License (http://creativecommons.org/licenses/by/2.0), which permits unrestricted use, distribution, and reproduction in any medium, provided the original work is properly cited. 
and then adapts the system parameters intelligently to achieve per-flow fairness or weighted fairness. We conduct simulations to evaluate the performance of the proposed adaptive algorithm. The simulation results demonstrate that our approach can effectively provide fairness of channel utilization between uplink and downlink in varying WLAN environments.

The remainder of this article is organized as follows. The following section presents some numerical results to illustrate the fairness problem and the related study. In Section "Proposed cross-layer adaptative algorithm", we illustrate our proposed adaptive control algorithm as a solution. In Section "Performance evaluations and results", we construct simulation scenarios to evaluate the effectiveness of the proposed scheme. Finally the article ends with conclusions.

\section{Unfairness problem and related work}

In this section, we conduct simulations to explore the fairness problem between uplink and downlink in IEEE 802.11 WLANs based a verified two-dimensional Markov chain model [3]. Figure 1 shows the transmission scenario where there are $N_{\mathrm{d}}$ mobile stations with downlink traffic and $N_{\mathrm{u}}$ mobile stations with uplink traffic in an infrastructure $802.11 \mathrm{~b}$ WLAN environment. Consider that each station processes a User-Datagram-Protocol traffic flow, and assume that all the transmissions are under ideal channel conditions using the highest data rate of 11 Mbps. Assume that all the flows always have packets to send (i.e., under a saturated condition). The adopted 802.11b parameters are shown in Table 1.

Figure 2 presents the aggregate throughput of downlink traffic and that of uplink traffic varying with the number of uplink stations, $N_{\mathrm{u}}$. It is shown that as the number of uplink station increases from 1 to 15 , the aggregate throughput of downlink traffic decrease from 3.364 to $0.396 \mathrm{Mbps}$, while that of uplink traffic increase

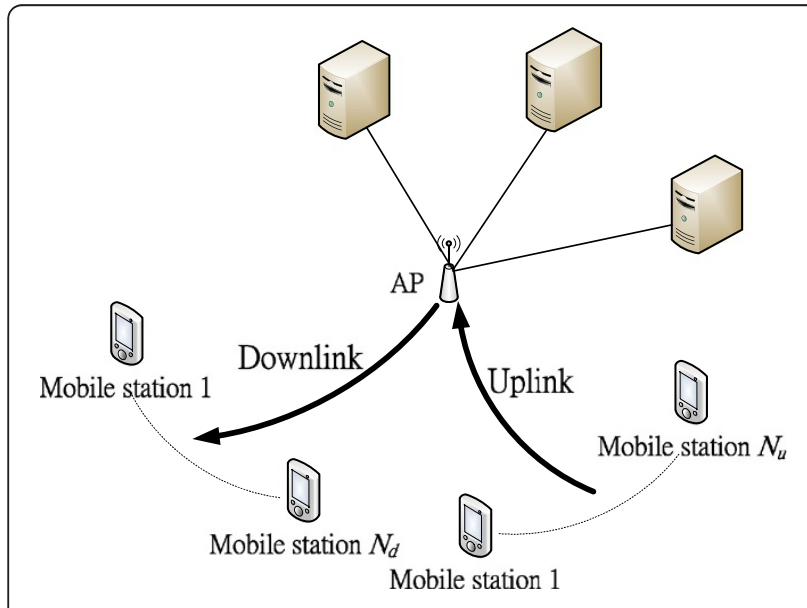

Figure 1 The downlink and uplink transmissions in WLANs.
Table 1 The adopted IEEE 802.11b parameter

\begin{tabular}{ll}
\hline Parameter & Value \\
\hline Transmission rate & 11 Mbps \\
Slot-time & $20 \mu \mathrm{s}$ \\
SIFS & $10 \mu \mathrm{s}$ \\
DIFS & $50 \mu \mathrm{s}$ \\
Payload & 1500 bytes \\
PHY header & 24 bytes \\
MAC header & 28 bytes \\
ACK frame & 38 bytes \\
CW ${ }_{\text {min }}$ & 32 \\
CW & 1024 \\
Retry limit & 5 \\
\hline
\end{tabular}

from 3.364 to 5.945 Mbps. We can observe that an increasing amount of uplink stations will reduce the transmission opportunity (TXOP) of downlink traffic and consequently the downlink throughput, while the downlink throughput is almost $1 / N_{\mathrm{d}}$ times the uplink throughput. It is due to the fact that the DCF protocol actually provides all 802.11 transmitting nodes including AP and each uplink station with the same TXOP, i.e., their channel-access probability is equal to $1 /\left(N_{\mathrm{u}}+1\right)$. However, AP is responsible for the deliveries of $N_{\mathrm{d}}$ downlink flows; therefore, the TXOP of one downlink flow is only $1 / N_{\mathrm{d}}$ times that of one uplink flow. It can introduce an unfair resource allocation between uplink and downlink, and this problem can particularly be critical when the amount of downlink flows increases or the traffic load of downlink is much heavier than that of uplink.

The fairness problems in IEEE 802.11 WLANs have largely been investigated in previous work [4-29]. The authors of [8] proposed to dynamically determine AP's minimum contention window size and TXOP limit according to the packet error rate and the number of stations. The study [9] presents a measurement-based

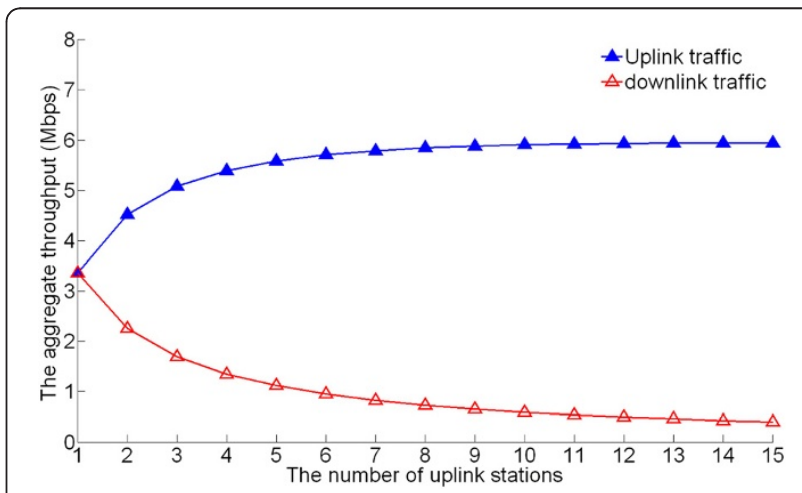

Figure 2 The aggregate throughput of uplink and downlink traffic varying with the number of uplink stations. 
adaptation algorithm which dynamically controls the enhanced distributed channel access parameter set to achieve a predetermined utilization ratio between uplink and downlink flows in 802.11e WLANs. The authors of [13] proposed an approach that reduces AP's channel sensing time from DCF inter-frame space (DIFS) to PCF inter-frame space (PIFS) in order to meet the required utilization ratio for downlink traffic flows. This approach grants AP the highest priority to transmit its data frames immediately, but may cause the entire channel slots occupied by AP before the required utilization ratio is matched. The study [14] presents a dynamic contention window control (DCWC) scheme based on the number of downlink flows to achieve per-flow fairness. Nevertheless, it does not consider some dynamics in WLAN environments such as channel conditions and traffic loads that can greatly impact the performance of fairness. The authors of [16] use an analytical approach to find optimal contention window sizes based on the observed idle slot intervals to achieve utility fairness between AP and wireless stations. However, the approaches proposed in [16] may need substantial modifications in the MAC layer protocols.

In general, the traffic load of downlink flows may be much heavier than that of uplink flows. The study in [21-23] investigates weighted fairness in case that the downlink and uplink traffic loads are asymmetric. The authors of [21] present the Bidirectional DCF which provides a preferential treatment to downlink traffic by piggybacking AP's data packets after acknowledge (ACK) frames. This approach can provide a ratio of downlink throughput to uplink throughput up to 1 . The study [22] developed adaptive schemes to achieve weighted fairness between uplink/downlink traffic flows by dynamically adjusting the backoff counters of AP and stations. The authors of [23] applied differentiated minimum contention windows $(\mathrm{CW})$ for $\mathrm{AP}$ and wireless stations to tune their channel utilization ratio.

The problem of transmission-control-protocol (TCP) unfairness in wireless networks has been researched in [24-29]. The study [24] provides a detail analysis of per- flow and per-station fairness for TCP flows. The authors of [25] proposed a differentiated approach which involves multidimensional parameters including minimum CWs, arbitration inter-frame space and TXOP, to solve the TCP fairness problem between uplink and downlink traffic flows in 802.11e WLANs. The authors of [27] propose a cross-layer feedback approach to achieve per-station fairness by estimating each station's access time and queue length. The study [28] solves the TCP fairness problem by using a dual queue scheme in which one queue is specified for data packets of downlink TCP flows and the other is for ACK packets.

\section{Proposed cross-layer adaptative algorithm}

In order to provide fair channel utilization between uplink and downlink, AP and mobile stations should be granted suitable transmission opportunities based on their bandwidth demands. In this article, we propose a cross-layer adaptive algorithm which dynamically adjusts the minimum CW of AP according to the dynamics of WLAN environments such as the numbers of traffic flows, channel conditions, and application-layer bandwidth demands to achieve both per-flow fairness and weighted fairness.

\section{Architecture of the proposed adaptive cross-layer algorithm}

Figure 3 shows the architecture of the proposed crosslayer approach. The architecture involves a throughput monitor at AP to periodically calculate the ratio between downlink and uplink throughputs. On the other hand, the optimal bandwidth sharing between uplink and downlink flows is determined by some external factors, including the situation of uplink/downlink traffic contentions, PHYlayer channel conditions, bandwidth demands of applications, etc. The reasons of considering these external factors for determining the bandwidth sharing are

(1) The channel utilization can be affected by both the amount of contending flows and channel conditions, i.e., BER

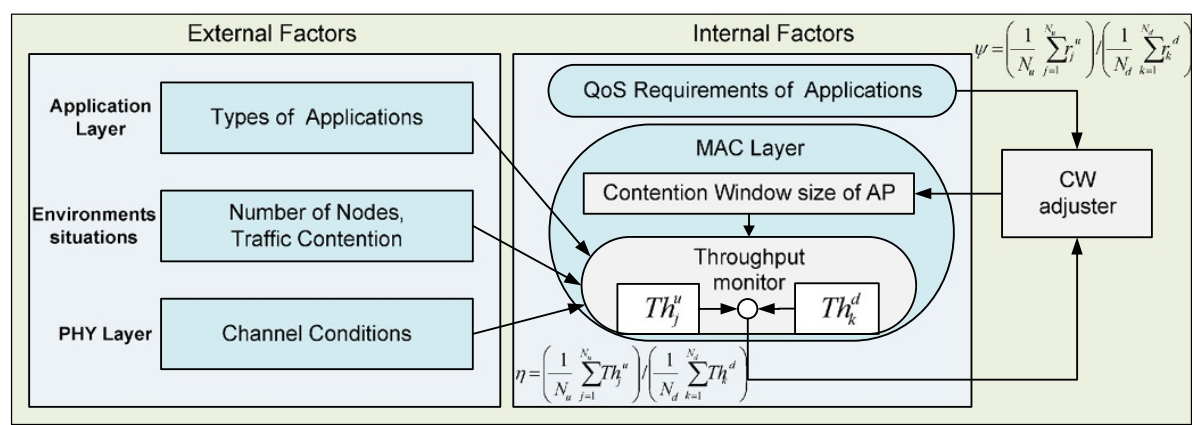

Figure 3 The architecture of the proposed cross-layer algorithm. 
(2) In case that uplink and downlink flows are with different bandwidth demands for various applications, the resources should be allocated based on their bandwidth requirements to provide weighted fairness.

Based on the changes of these external factors (environmental contexts and cross-layer impacts), our scheme will therefore adaptively adjust the internal factors (i.e., MAC parameters) to achieve per-flow fairness or weighted fairness. The 802.11 channel utilization in fact can be affected by many MAC parameters, e.g., inter-frame space (IFS), minimum CW, and TXOP. In this article, we adopt the parameter of minimum $\mathrm{CW}\left(\mathrm{CW}_{\min }\right)$ for the proposed adaptation scheme since it is a key parameter affecting not only the access priority but also the overall system performance [30].

\section{Contention window adjuster}

We design a contention window adjuster (CWA) based on a feedback control mechanism to adaptively adjust $\mathrm{CW}_{\text {min }}$ toward the optimal value. Figure 4 shows the feedback control mechanism of CWA. There are two context metrics for CWA. One metric is the ratio of average uplink throughput to average downlink throughput, $\eta$. It can be determined by means of a measurement-based approach at the AP site. The metric $\eta$ is defined as

$$
\eta=\left(\frac{1}{N_{\mathrm{u}}} \sum_{j=1}^{N_{\mathrm{u}}} T h_{j}{ }^{u}\right) /\left(\frac{1}{N_{\mathrm{d}}} \sum_{k=1}^{N_{\mathrm{d}}} T h_{k}{ }^{d}\right),
$$

where $N_{\mathrm{u}}$ and $N_{\mathrm{d}}$ are the number of uplink and downlink flows, respectively; $T h_{j}^{u}$ and $T h_{k}^{d}$ is the throughput of uplink flow $j$ and downlink flow $k$, respectively, measured at AP periodically.

\section{Another context metric is the ratio of average uplink} bandwidth requirements to average downlink bandwidth requirements, $\psi$. The metric $\psi$ is defined as

$$
\psi=\left(\frac{1}{N_{\mathrm{u}}} \sum_{j=1}^{N_{\mathrm{u}}} r_{j}^{u}\right) /\left(\frac{1}{N_{\mathrm{d}}} \sum_{k=1}^{N_{\mathrm{d}}} r_{k}^{d}\right),
$$

where $r_{j}^{u}$ and $r_{k}^{d}$ is the bandwidth requirement of uplink flow $j$ and downlink flow $k$, respectively. This context can be obtained by packet exchanges between mobile stations and AP. For example, mobile stations can periodically advertise AP of their bandwidth demands with a piggy-back technique using ACK frame.

With the two information metrics, $\eta$ and $\psi$, CWA will therefore adaptively adjust $\mathrm{CW}_{\min }$ such that $\psi / \eta$ is equal to 1 to provide per-flow fairness or weighted fairness, depending on the value of $\psi$. For example, if $\psi$ is equal to 1, i.e., uplink and downlink flows are with the same bandwidth requirements, CWA will adaptively adjust $\mathrm{CW}_{\min }$ such that $\eta$ is equal to 1 to provide per-flow fairness. Alternatively, if $\psi$ is an arbitrary number larger or smaller than 1 when uplink and downlink flows are with different bandwidth demands, CWA will adjust $\mathrm{CW}_{\text {min }}$ according to users' requirements such that $\eta$ is equal to $\psi$ to provide weighted fairness.

CWA iteratively adjusts $\mathrm{CW}_{\min }$ in order to have the value of $\psi / \eta$ as close to 1 as possible. The iterative formula to adjust $\mathrm{CW}_{\min }$ is

$$
\begin{aligned}
& C W_{\min , i+1}=C W_{\min , i}+\Delta_{i} \\
& \Delta_{i}=A \log _{2}\left(\psi_{i} / \eta_{i}\right),
\end{aligned}
$$

where $\mathrm{CW}_{\min , i}$ is the $\mathrm{CW}_{\min }$ of $i$ th adaptation; $\Delta_{i}$ is $i$ th adaptation step size; $A$ is the normalized step size $(A>0)$. From Equation (4), we can see that the adaptation step size $\Delta_{i}$ depends on $A, \eta_{i}$ and $\psi_{i}$. If $\psi_{i} / \eta_{i}$ is far from $1, \Delta_{i}$ will be larger; when $\psi_{i} / \eta_{i}$ is close to $1, \Delta_{i}$ becomes smaller. Note that the adaptation mechanism can work well to provide fairness in both cases when $\psi_{i} /$ $\eta_{i}$ is larger or smaller than 1 . In general cases when $\psi_{i} / \eta_{i}$ is smaller than 1 , the step size $\Delta_{i}$ will be positive to decrease $\mathrm{CW}_{\text {min }}$. It is noticeable that although $\Delta_{i}$ can be an arbitrary real number, $\mathrm{CW}_{\min }$ must be a positive integer. Thus, the chosen value for $\mathrm{CW}_{\min }$ will be rounded to the closest integer. Alternatively, when $\psi_{i} / \eta_{i}$ is larger than 1, e.g., the amount of uplink flows is rare or bandwidth requirements of downlink are less than that of uplink, $\Delta_{i}$ will be negative to increase $C W_{\text {min }}$. After several adaptation steps when $\psi_{i} / \eta_{i}$ is close to $1, \Delta_{i}$ will be rather small or even zero, and consequently $\mathrm{CW}_{\text {min }}$ will almost keep steady. At this time the adaptation of $\mathrm{CW}_{\text {min }}$

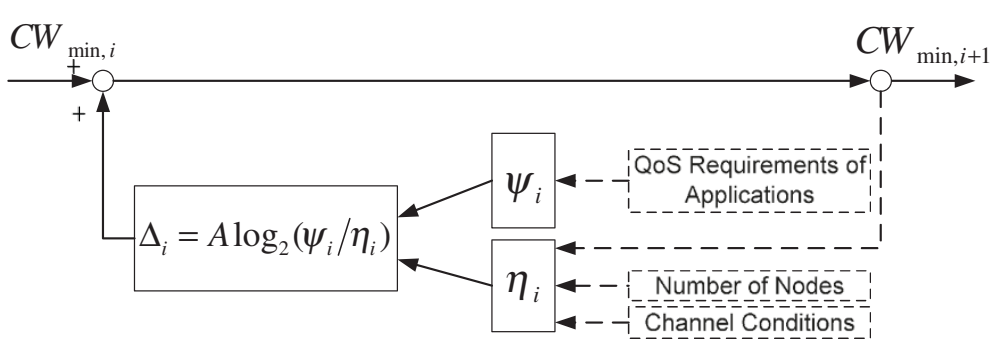

Figure 4 The CWA. 
converges to the optimal value in terms of the best performance of fairness.

Note that the value of $\psi_{i} / \eta_{i}$ can vary throughout the whole transmission period due to the dynamics of WLAN environments such as channel conditions, numbers of traffic flows, application-layer bandwidth demands, etc. When $\psi_{i} / \eta_{i}$ is removed from the target value of 1 due to the change of WLAN environments, the proposed feedback control mechanism aforementioned can automatically tune $\mathrm{CW}_{\min }$ to the optimal value with regard to the current situations.

To evaluate the performance of fairness between uplink and downlink, we use the Jain fairness index [18]. It is a pertinent criterion index for the fairness of channel utilization in contending 802.11 WLANs. The Jain fairness index, $\Gamma$, in this study can be represented as

$$
\Gamma=\frac{\left(\sum_{j=1}^{N_{u}} \frac{T h_{j}^{u}}{r_{j}^{u}}+\sum_{k=1}^{N_{d}} \frac{T h_{k}^{d}}{r_{k}^{d}}\right)^{2}}{\left(N_{u}+N_{d}\right)\left[\sum_{j=1}^{N_{u}}\left(\frac{T h_{j}^{u}}{r_{j}^{u}}\right)^{2}+\sum_{k=1}^{N_{d}}\left(\frac{T h_{k}^{d}}{r_{k}^{d}}\right)^{2}\right]}
$$

$\Gamma$ has a range of $(0,1]$ to evaluate the fairness; the value closer to 1 refers to better performances of fairness. The index shown in Equation (5) can be used to assess both the per-flow fairness and weighted fairness.

\section{Performance evaluations and results}

In this section, we conduct simulations of an IEEE 802.11 transmission scenario to estimate the performance of the proposed algorithm. From the simulation results, we demonstrate the effectiveness of our algorithm to provide per-flow fairness between downlink and uplink traffics, and further to provide weighted fairness according to users' bandwidth requirements. The IEEE 802.11 simulation model was built based on our analytical approach [31] which has been developed by extending a verified two-dimensional Markov chain model proposed by Bianchi [3]. However, our analytical model takes into account more realistic factors, including error-prone channels, multiple data rates, the finite retransmission limit, etc. Thus, our approach could be more practical to provide performance evaluations of 802.11 DCF in realistic WLAN environments. The restriction of this model is that it considers only saturated traffic (i.e., all the flows always have packets to send), and that it does not take into account the capture effect (i.e., all the data transmissions will fail in the presence of packet collisions without the consideration of their relative signal strength). We wrote Matlab codes to implement the IEEE 802.11 model and provide numerical results in the simulations. The adopted $802.11 \mathrm{~b}$ parameters are shown in Table 1. In our adaptive scheme, the normalized step size $A$ in Equation (4) is set as 2 . We compare the performance of the proposed adaptive control algorithm with that of IEEE 802.11 DCF protocol [1], and in some scenarios further with that of the DCWC scheme [14]. The DCWC scheme determines the optimal value of minimum $\mathrm{CW}$ of $\mathrm{AP}$ to achieve per-flow fairness according to the number of downlink flows. The performances are indexed as uplink/downlink throughputs and the Jain fairness index.

\section{Scenario I: equal bandwidth requirements under ideal channel conditions}

The simulation set-up for this scenario assumes 12 downlink and 8 uplink flows of the same class with similar QoS requirements under ideal channel environments. Figure 5 shows per-flow throughput of uplink and downlink with 802.11 DCF and the proposed adaptation scheme, respectively. Figure 5 shows that the downlink and uplink throughputs with 802.11 DCF are 60 and 730 kbps, respectively; the bandwidth sharing between uplink and downlink is quite unfair. To achieve fairness, more resources should be taken from uplink flows and then allocated to downlink flows. With the proposed adaptive scheme, the bandwidth sharing between uplink and downlink is regulated by adapting the parameter of $\mathrm{CW}_{\text {min }}$. Figure 5 also shows that the downlink throughput can gradually approach the level of $310 \mathrm{kbps}$ at the eighth adaptation sequence while the uplink throughput comes to $360 \mathrm{kbps}$ nearby; the fairness of bandwidth sharing between uplink and downlink is greatly improved.

Figure 6 presents the Jain utility fairness index $\Gamma$ with 802.11 DCF and the proposed scheme, respectively. It is shown that $\Gamma$ with DCF is $50.1 \%$ steadily. With our scheme, $\Gamma$ rises and reaches the level of $99.5 \%$ (1 refers to the best fairness) at the eighth adaptation sequence and the throughput levels of uplink and downlink flows are rather close at this moment (refer to Figure 5). The

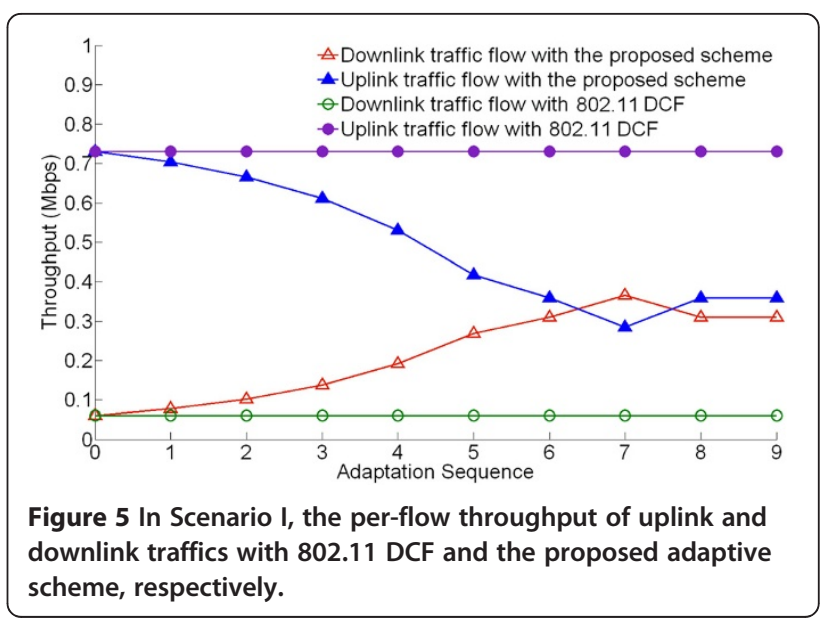




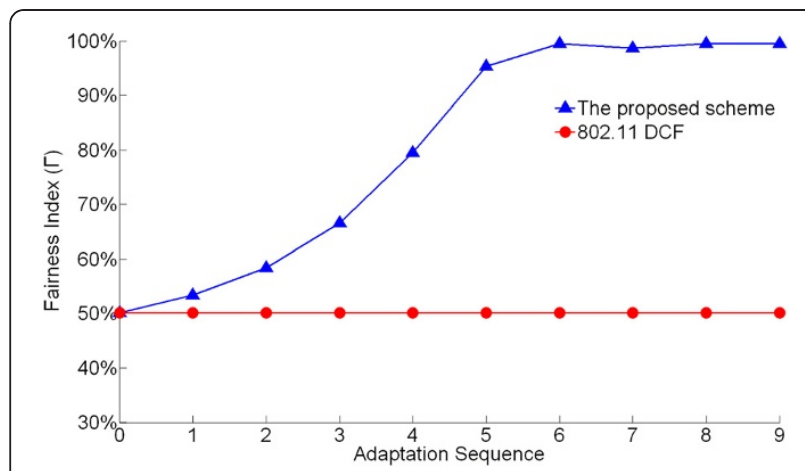

Figure 6 In Scenario I, the Jain fairness index with 802.11 DCF and the proposed adaptive scheme, respectively.

results demonstrate that our cross-layer approach can effectively provide per-flow fairness between uplink and downlink in WLAN environments.

\section{Scenario II: dynamic flow amounts in the network}

In this scenario, we assume eight downlink flows and eight uplink flows in the network initially and two more downlink flows joining in later. Figure 7 presents perflow throughput of uplink and downlink with 802.11 DCF and the proposed adaptation scheme, respectively. With 802.11 DCF, the uplink throughput is $730 \mathrm{kbps}$ steadily, while the downlink throughput is $91 \mathrm{kbps}$ initially and degrades to $73 \mathrm{kbps}$ when two more downlink flows joins in the network at the adaptation sequence of 12. The unfairness of bandwidth sharing between uplink and downlink becomes severer when the number of downlink flows increases. With the proposed scheme, it is shown in Figure 7 that the uplink and downlink throughputs converge to the level of about $410 \mathrm{kbps}$ at the nineth adaptation sequence. When two more downlink flows join in the network later (at the 12th adaptation sequence), the value of $\mathrm{CW}_{\min }$, which achieves fair utility earlier ceases to be effective at this moment. With

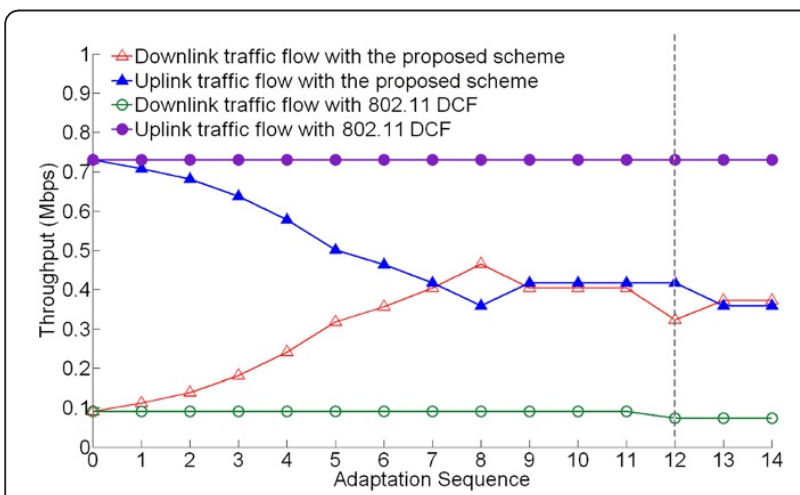

Figure 7 In Scenario II, the per-flow throughput of uplink and downlink traffics with 802.11 DCF and the proposed adaptive scheme, respectively. our adaptive mechanism sensing the change of WLAN conditions (i.e., the number of downlink flows) and adjusting $\mathrm{CW}_{\min }$ accordingly, the uplink and downlink throughputs approach each other again (about $366 \mathrm{kbps}$ ) at the sequence of 13 .

Figure 8 shows the Jain utility fairness index $\Gamma$ with 802.11 DCF and the proposed scheme, respectively. It is shown that $\Gamma$ with DCF is $62.3 \%$ initially and degrades to $55.6 \%$ when two more downlink flows joins in the network. With our scheme, it is shown that $\Gamma$ rises and reaches the level of $99.9 \%$ at thenineth adaptation sequence when the uplink and downlink throughputs are quite near (about $410 \mathrm{kbps}$ ) at this moment (refer to Figure 7). Later when two more downlink flows joins in the network, $\Gamma$ slightly drops to $98.4 \%$ since the difference between uplink and downlink throughputs is enlarged. It is shown that $\Gamma$ rises to $99.9 \%$ at the 14 th sequence while the uplink and downlink throughputs are almost equal again (about $366 \mathrm{kbps}$ ) at this moment. The results demonstrate that our approach can sense the changes of WLAN environments (i.e., the number of traffic flows) and adjust system parameters accordingly to provide fair utilities between uplink and downlink.

\section{Scenario III: equal bandwidth requirements under diverse and time-varying channel conditions}

The simulation set-up for this scenario considers ten downlink flows and ten uplink flows with diverse and time-varying channel conditions. Assume that the uplink flows are in ideal channel conditions (i.e., BER is 0 ), whereas the downlink flows are with worse link qualities with BER of 5E-6 initially, and later suffer from channel degradation with BER of $1.5 \mathrm{E}-5$. Figure 9 presents perflow throughput of uplink and downlink with 802.11 DCF and the proposed adaptation scheme, respectively. With 802.11 DCF, the downlink (uplink) throughput is $50 \mathrm{kbps}$ (597 kbps) initially and decreases (increases) to $35 \mathrm{kbps}(609 \mathrm{kbps})$ as the communication environment deteriorates. As shown in Figure 10, the Jain utility

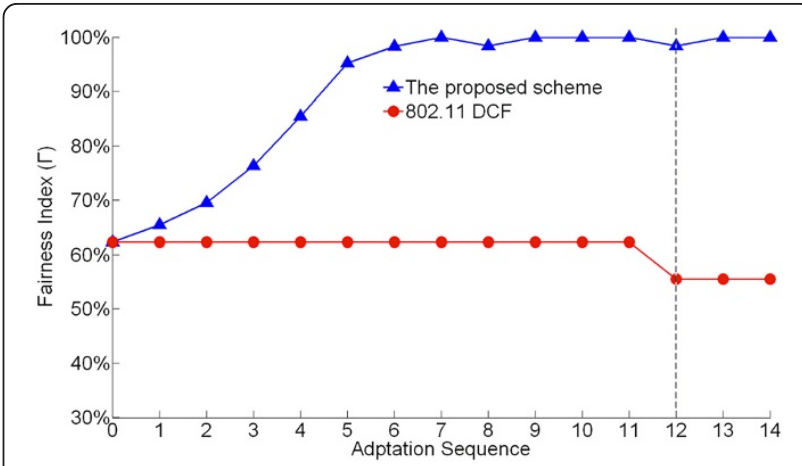

Figure 8 In Scenario II, the Jain fairness index with 802.11 DCF and the proposed adaptive scheme, respectively. 


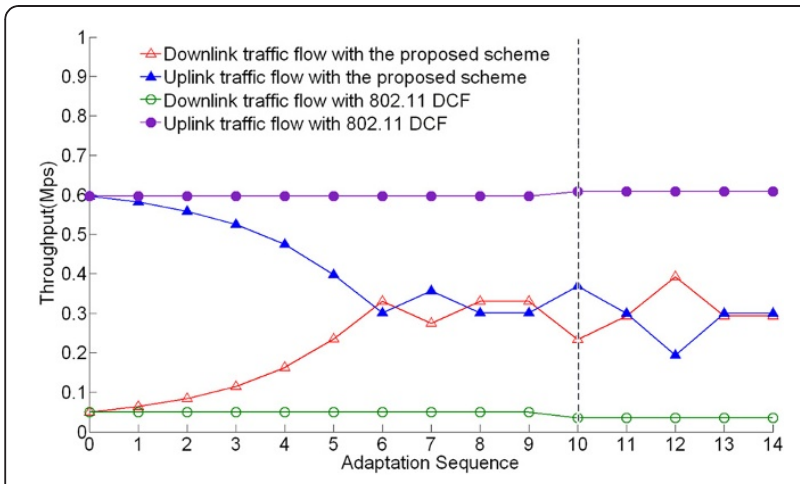

Figure 9 In Scenario III, the per-flow throughput of uplink and downlink traffics with $802.11 \mathrm{DCF}$ and the proposed adaptive scheme, respectively.

fairness index $\Gamma$ decreases from 58.3 to $55.7 \%$ in the corresponding time. The degradation of utility fairness between uplink and downlink flows is due to their diverse channel conditions.

With the proposed adaptation scheme, the fairness of channel sharing is improved significantly. The downlink throughput progressively increases and reaches $331 \mathrm{kbps}$ at the eighth adaptation sequence while the uplink throughput comes to a similar level (301 kbps) at this time $(\Gamma=99.8 \%$ as shown in Figure 10$)$. When the link quality of downlink flows deteriorates later at the adaptation sequence of 10 , the value of $\mathrm{CW}_{\min }$ adjusted earlier cease to be effective in the current situation. Consequently the downlink throughput drops to 233 kbps whereas the uplink throughput increases to 368 kbps; the variation between uplink and downlink throughput increases noticeably $(\Gamma=95.2 \%)$. With our scheme which adaptively adjusts $\mathrm{CW}_{\text {min }}$ regarding the channel diversity of traffic flows, the uplink and downlink throughputs are almost equal (296 kbps) again after the sequence of 13 ( $\Gamma=99.9 \%)$. The results demonstrate that our adaptation scheme can effectively tackle a

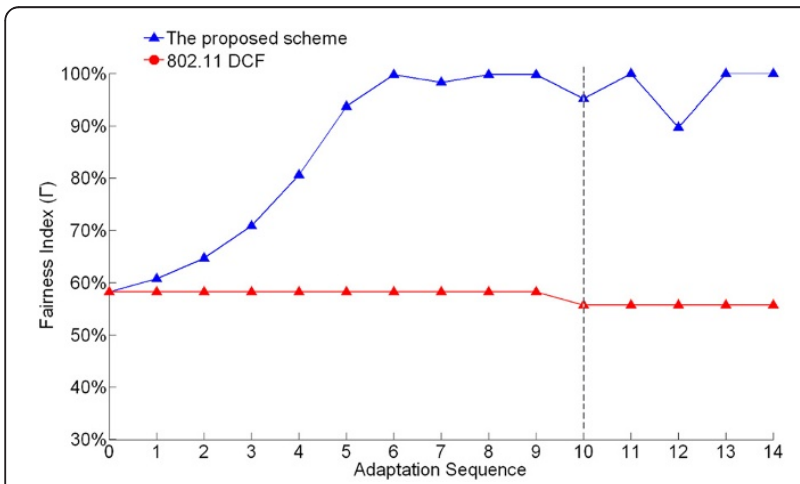

Figure 10 In Scenario III, the Jain fairness index with $\mathbf{8 0 2 . 1 1}$ DCF and the proposed adaptive scheme, respectively. variety of channel conditions to provide fair channel utilization between uplink and downlink.

\section{Scenario IV: comparison with the DCWC scheme}

In the section, we compare the performance of the proposed scheme with that of the DCWC scheme [14]. The simulation scenario is similar to that in the previous section (Scenario III), except that the link qualities of downlink flows are ideal initially, become worse later with BER of $5 \mathrm{E}-6$, and finally deteriorate with BER of $1.5 \mathrm{E}-5$. Figure 11 presents per-flow throughput of uplink and downlink with the DCWC scheme and our proposed scheme, respectively. With the DCWC scheme, the downlink (uplink) throughput is $323 \mathrm{kbps}$ (327 kbps) initially and comes to $274 \mathrm{kbps}$ ( $357 \mathrm{kbps}$ ) and $233 \mathrm{kbps}$ (368 kbps) successively as channel conditions change at the adaptation sequence of 10 and 13 sequentially. It is shown that the difference of throughput between uplink and downlink flows sharply increases from $4 \mathrm{kbps}(1 \%)$ to $83 \mathrm{kbps}(30 \%)$ and $135 \mathrm{kbps}(58 \%)$ sequentially as the communication environment deteriorates. We can observe that the DCWC scheme performs well to provide fairness with ideal link qualities, but may be ineffective under varying and diverse channel conditions. The degradation of fairness performance with the DCWC scheme is posed by a skewed channel sharing due to using fixed parameters in varying channel conditions.

With the proposed scheme, the fairness of channel sharing between uplink and downlink can gradually be achieved. The downlink (uplink) throughput comes to $323 \mathrm{kbps}$ (327 kbps) at the adaptation sequence of 8, 330 kbps (301 kbps) at the sequence of 11, and $293 \mathrm{kbps}$ (300 kbps) at the sequence of 16. The variation of throughput between uplink and downlink flows corresponding to these moments is quite small as $4 \mathrm{kbps}$ (1\%), $29 \mathrm{kbps}(10 \%)$, and $7 \mathrm{kbps}(2 \%)$, respectively. The performance difference between the DCWC scheme and our proposed adaptation scheme can be illustrated with

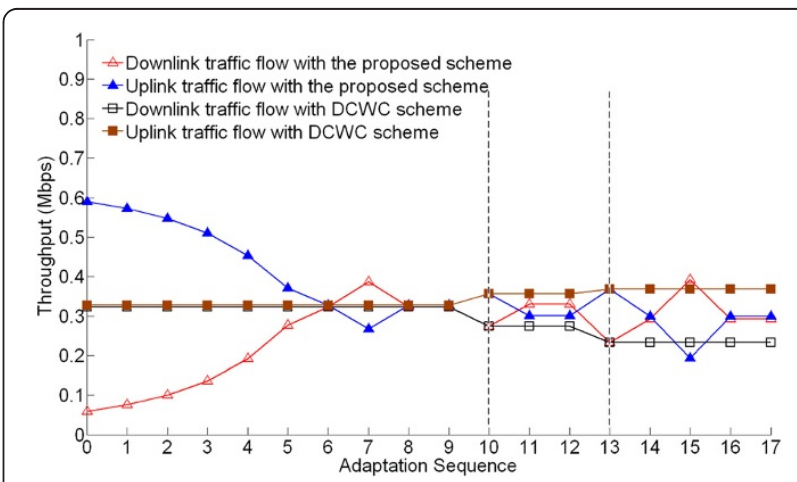

Figure 11 In Scenario IV, the per-flow throughput of uplink and downlink traffics with the DCWC scheme and our proposed adaptive scheme, respectively. 
their adaptation trajectories of $\mathrm{CW}_{\text {min }}$ as shown in Figure 12. We can observe that the DCWC scheme adopts the constant value of 5 as the optimal value for $\mathrm{CW}_{\text {min }}$, no matter how the channel environment may vary. In contrast, our scheme adaptively tunes $\mathrm{CW}_{\min }$ with regard to channel conditions, and it is shown that the optimal value change from 5 to 4 and 3 consecutively in different channel situations.

The performance difference can also be clearly shown in Figure 13 which presents the Jain utility fairness index $\Gamma$ with the DCWC scheme and the proposed scheme, respectively. It is shown that $\Gamma$ with the DCWC scheme decreases from 99.9 to $98.3 \%$ and $88.2 \%$ sequentially as the communication environment deteriorates. With our scheme, it is shown that $\Gamma$ converges to $99.9,99.8$, and $99.9 \%$ sequentially at the adaptation sequence of 8,11 , and 16 , respectively. The results demonstrate that our cross-layer approach can sense the change of WLAN environments (i.e., channel conditions) and adjust system parameters accordingly to achieve fairness under varying WLAN environments.

\section{Scenario V: diverse bandwidth requirements}

Finally, we present the simulation results which demonstrate that our scheme can effectively provide weighted fairness according to users' bandwidth requirements. The simulation set-up for this scenario considers eight downlink flows and eight uplink flows under ideal channel conditions, and assumes that the bandwidth requirement of a downlink flow is two times that of an uplink flow. Figure 14 presents per-flow throughput for uplink and downlink with 802.11 DCF and the proposed adaptation scheme, respectively. It is shown that with 802.11 DCF, the downlink throughput is $91 \mathrm{kbps}$ while the uplink throughput is $731 \mathrm{kbps}$; the ratio of downlink throughput to uplink throughput is about 1:8 (the corresponding $\Gamma=56.2 \%$ as shown in Figure 15), which is far

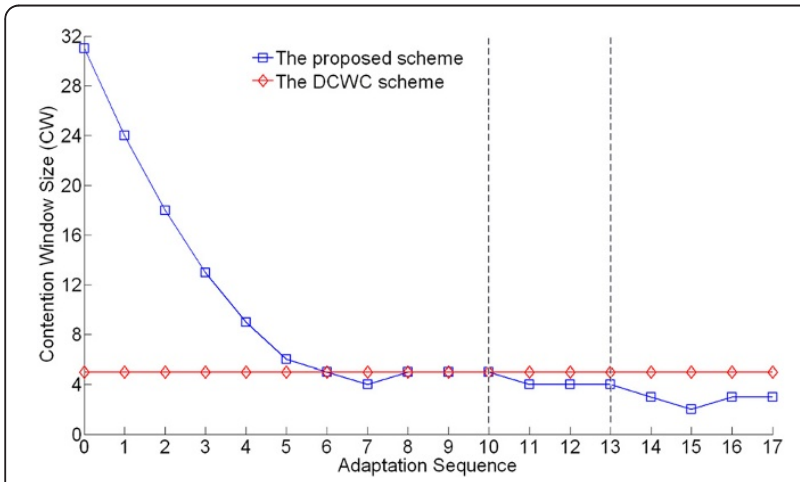

Figure 12 In Scenario IV, the adaptation trajectory of $\mathrm{CW}_{\min }$ with the DCWC scheme and our proposed adaptive scheme, respectively.

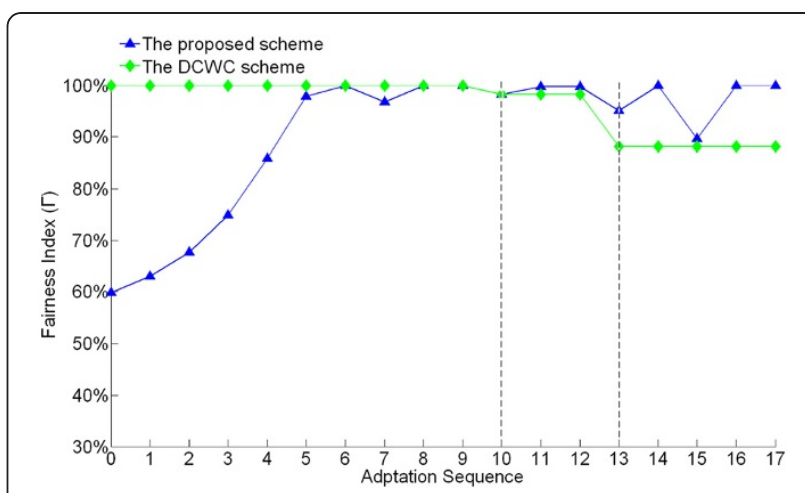

Figure 13 In Scenario IV, the Jain fairness index with the DCWC scheme and our proposed adaptive scheme, respectively.

from the target value 2:1 aforementioned. With the proposed scheme, the downlink throughput gradually increases and converges to $548 \mathrm{kbps}$ at the seventh adaptation sequence, while the uplink throughput gradually decreases and comes to $285 \mathrm{kbps}$; the ratio of downlink throughput to uplink throughput is $1.92: 1$ ( $\Gamma=99.9 \%)$, which can nearly meet the desired bandwidth demands between downlink and uplink, 2:1. The results demonstrate that our adaptation scheme can effectively provide weighted fairness between uplink and downlink according to users' bandwidth requirements in WLAN environments.

\section{Conclusion}

In this article, we investigate a fairness issue between uplink and downlink flows in IEEE 802.11 WLANs. We propose a cross-layer adaptive algorithm to achieve both per-flow fairness and weighted fairness based on a feedback control mechanism which dynamically adjusts the contention window size of AP according to the dynamics of WLAN environments such as the numbers of traffic

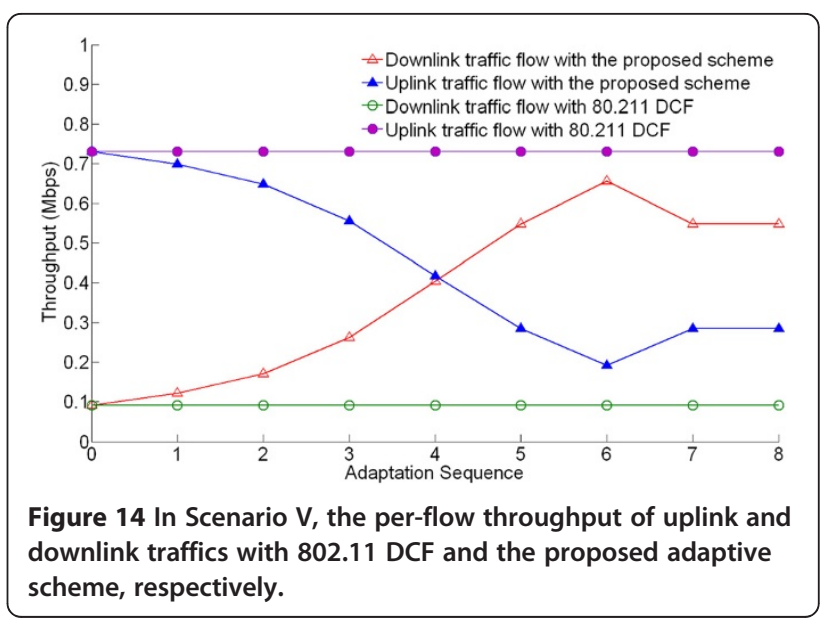




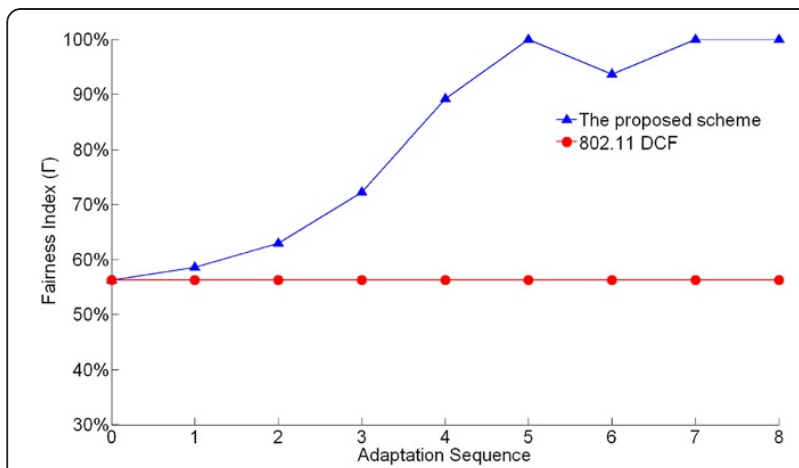

Figure $\mathbf{1 5}$ In Scenario V, the Jain fairness index with $\mathbf{8 0 2 . 1 1}$ DCF and the proposed adaptive scheme, respectively.

flows, channel conditions, application-layer bandwidth demands, etc. The simulation results demonstrate that our scheme can effectively provide both per-flow fairness and weighted fairness in a varying WLAN environment.

The proposed cross-layer algorithm has to comprehend the application layer information, i.e., users' bandwidth demands for the provision of weighted fairness. Thus it is required for our algorithm to implement a mechanism which governs the exchange of application-layer contexts between mobile stations and AP. We will keep this issue as future work.

\section{Competing interests}

The authors declare that they have no competing interests.

\section{Acknowledgements}

This work was supported in part by Taiwan National Science Council under Grant 99-2221-E-003-005 and 100-2221-E-003-020.

Received: 13 February 2012 Accepted: 12 July 2012

Published: 31 July 2012

\section{References}

1. IEEE 802.11a/b, Wireless LAN Medium Access Control (MAC) and Physical Layer (PHY) Specifications, Standard, IEEE (1999)

2. IEEE 802.11e, Wireless LAN MAC PHY: Medium Access Control (MAC) Characterof-Service Enhancements, IEEE (2005)

3. G. Bianchi, Performance analysis of the IEEE 802.11 distributed coordination function. IEEE J. Sel. Areas Commun. 18(3), 535-547 (2000)

4. W. Li, Y. Cui, X. Cheng, M.A. Al-Rodhaan, A. Al-Dhelaan, Achieving proportional fairness via AP power control in multi-rate WLANs. IEEE Trans. Wirel. Commun. 10(11), 3784-3792 (2011)

5. S. Chun, D. Xianhua, L. Pingyuan, Z. Han, Adaptive access mechanism with optimal contention window based on node number estimation using multiple thresholds. IEEE Trans. Wirel. Commun. 10(11), 3784-3792 (2011)

6. S.K. Dandapat, B. Mitra, R.R. Choudhury, N. Ganguly, Smart association control in wireless mobile environment using max-flow. IEEE Trans. Netw. Serv. Manag. 9(1), 73-86 (2012)

7. Y. Le, L. Ma, W. Cheng, X. Cheng, B. Chen, Maximizing throughput when achieving time fairness in multi-rate wireless LANs, in Proc. of IEEE INFOCOM Mini-Conference (Orlando, Florida, USA, 2012), pp. 2911-2915

8. W.-S. Lim, D.-W. Kim, Y.-J. Suh, Achieving fairness between uplink and downlink flows in error-prone WLANs. IEEE Commun. Lett. 15(8), 822-824 (2011)

9. F. Keceli, I. Inan, E. Ayanoglu, Weighted fair uplink/downlink access provisioning in IEEE 802.11e WLANs, in Proc. of IEEE International Conference on Communications (Beijing, China, 2008), pp. 2473-2479

10. Z. Kong, D.H.K. Tsang, B. Bensaou, D. Gao, Performance analysis of the IEEE 802.11e contention-based channel access. IEEE J. Sel. Areas Commun. 22(10), 2095-2106 (2004)
11. Y. Xiao, Performance analysis of priority schemes for IEEE 802.11 and IEEE 802.11e wireless LANs. IEEE Trans. Wirel. Commun. 4(4), 1506-1515 (2005)

12. I. Inan, F. Keceli, E. Ayanoglu, Analysis of the 802.11e enhanced distributed channel access function. IEEE Trans. Commun. 57(6), 1753-1764 (2009)

13. S.W. Kim, B.-S. Kim, Y. Fang, Downlink and uplink resource allocation in IEEE 802.11 wireless LANs. IEEE Trans. Veh. Technol. 54(1), 320-327 (2005)

14. B.A.H.S. Abeysekera, T. Matsuda, T. Takine, Dynamic contention window control to achieve fairness between uplink and downlink flows in IEEE 802.11 WLANs, in Proc. of IEEE Wireless Communications and Networking Conference (Hong Kong, China, 2007), pp. 2109-2114

15. B.A.H.S. Abeysekera, T. Matsuda, T. Takine, Dynamic contention window control mechanism to achieve fairness between uplink and downlink flows in IEEE 802.11 wireless LANs. IEEE Trans. Mob. Comput. 7(9), 3517-3525 (2008)

16. M. Heusse, F. Rousseau, R. Guillier, A. Duda, Idle sense: an optimal access method for high throughput and fairness in rate changeful wireless LANs, in Proc. of ACM SIGCOMM '05, vol. 35, August 2005 (Philadelphia, PA, USA, ), pp. 121-132

17. E. L-Aquilera, M. Heusse, Y. Grunenberger, F. Rousseau, A. Duda, J. Casademont, An asymmetric access point for solving the unfairness problem in WLANs. IEEE Trans. Mob. Comput. 7(10), 1213-1227 (2008)

18. D. Pong, T. Moors, Fairness and capacity trade-off in IEEE 802.11 WLANs, in Proc. of IEEE Conference on Local Computer Networks (Florida, USA, 2004), pp. 310-317

19. A. Trunganont, V. Visoottiviseth, Adaptive wireless bandwidth allocation for per-station fairness, in Proc. of International Symposium on Communications and Information Technology (ISCIT) (Incheon, Korea, 2009), pp. 1286-1291

20. M. Bredel, M. Fidler, Understanding fairness and its impact on quality of service in IEEE 802.11, in Proc. of IEEE INFOCOM (, Rio de Janeiro, Brazil, 2009), pp. 1098-1106

21. N.S.P. Nandiraju, H. Gossain, D. Cavalcanti, K.R. Chowdhury, D.P. Agrawa, Achieving fairness in wireless LANs by enhanced IEEE 802.11 DCF, in Proc. of IEEE Conference on Wireless and Mobile Computing, Networking and Communications (WiMob) (Montreal, Canada, 2006), pp. 132-139

22. J. Jeong, S. Choi, C.-K. Kim, Achieving weighted fairness between uplink and downlink in IEEE 802.11DCF-based WLANs, in Proc. of 2nd ACM International Conference on Quality of Service in Heterogeneous Wired/ Wireless Networks (QShine) (, Lake Buena Vista, Florida, USA, 2005)

23. M. Bottigliengo, C. Casetti, C.F. Chiasserini, M. Meo, Smart traffic scheduling in 802.11 WLANs with access point, in Proc. of IEEE VTC-Fall (Orlando, Florida, USA, 2003), pp. 2227-2231

24. M. Seyedzadegan, M. Othman, S. Subramaniam, Z. Zukarnain, The TCP fairness in WLAN: a review, in Proc. of IEEE Telecommunications and Malaysia International Conference on Communication (Penang, Malaysia, 2007), pp. 644-648

25. D.J. Leith, P. Clifford, D. Malone, A. Ng, TCP fairness in 802.11e WLANs. IEEE Commun. Lett. 9(11), 964-966 (2005)

26. N. Khademi, M. Othman, Guaranteeing per station and per flow fairness of downstream and upstream flows over IEEE 802.11 WLAN, in Proc. of International Conference on Information and Multimedia Technology (ICIMT) (Jeju Island, South Korea, 2009), pp. 431-435

27. E.-C. Park, D.-Y. Kim, H. Kim, C.-H. Choi, A cross-layer approach for per-station fairness in TCP over WLANs. IEEE Trans. Mob. Comput. 7(7), 898-910 (2008)

28. J. Ha, C.-H. Choi, TCP fairness for uplink and downlink flows in WLANs, in Proc. of IEEE Globecom'06 (San Francisco, California, USA, 2006)

29. Y. Wu, Z. Niu, J. Zheng, Study of the TCP upstream/downstream unfairness issue with per-flow queuing over infrastructure-mode WLANs. Wirel. Commun. Mob. Comput. 5(4), 459-471 (2005)

30. I. Tinnirello, G. Bianchi, L. Scalia, Performance evaluation of differentiated access mechanisms effectiveness in 802.11 networks, in Proc. of IEEE Globecom'04 (Dallas, Texas, USA, 2004), pp. 3007-3011

31. C. Wang, H.-K. Lo, S.-H. Fang, Fairness analysis of throughput and delay in WLAN environments with channel diversities. EURASIP J. Wirel. Commun. Netw. 201(1), 42 (2011)

doi:10.1186/1687-1499-2012-239

Cite this article as: Wang: Achieving per-flow and weighted fairness for uplink and downlink in IEEE 802.11 WLANs. EURASIP Journal on Wireless Communications and Networking 2012 2012:239. 